\title{
Solitary wave in a Burridge-Knopoff model with slip-dependent friction as a clue to understanding the mechanism of the self-healing slip pulse in an earthquake rupture process
}

\author{
Z. L. Wu and Y. T. Chen \\ Institute of Geophysics. China Seismological Bureau, 100081 Beijing, China
}

Received: 7 October 1996 - Revised: 19 July 1998 - Accepted: 14 September 1998

\section{Abstract}

A one-dimensional Burridge-Knopoff spring-block model with slip-dependent friction was studied to explore the possibility of a solitary wave solution existing for the problem of earthquake faulting. The result may be used as an alternative case of the crack model (e.g., Madariaga and Cochard, 1996) and the spring-block model with velocitydependent friction (e.g., Carlson and Langer, 1989) in the understanding of the mechanism of the self-healing slip pulse proposed by Heaton (1990). In general, the conditions for a solitary wave solution to exist are discussed by the trajectory in the phase space. By taking the first order approximation, it is demonstrated that a solitary wave solution exists in which the slip behaves as a propagating solitary wave with the propagation velocity less than that of an acoustic (seismic) wave, and the source time function at each position remains the same. As an alternative approach other than numerical calculations, the analytical solution, although simple, sheds light on some of the properties of the self-healing slip pulse. From the solution it is seen that the width of the pulse depends on its propagation velocity and the friction, consistent with experience in physics. It is pointed out that the self-healing slip pulse may exist for a broad class of frictional constitutive laws which, to some extent, explains the fact that the self-healing slip pulse may be observed for a variety of earthquakes occurring within different seismogenic environments.

\section{Introduction}

Heaton (1990) proposed that earthquake rupture occurs in a narrow self-healing pulse of slip that travels along the fault surface, which is supported by the accumulating results of the rupture process of earthquakes (e.g., Wald and Heaton, 1994; Irikura et al., 1996; Ide and Takeo, 1997; Nakayama and Takeo, 1997; Xu et al., 1997). The mechanism of this phenomenon is still under discussion, while most seismologists agree with the idea of Heaton (1990) that the mechanism of the self-healing slip pulse depends mainly on the constitutive law of friction, or more exactly, the coupling between the two walls of the earthquake fault (for simplicity we use the word 'friction' in this sense hereafter, but keep in mind that such 'friction' includes both the real friction in the pure mechanical sense and the apparent 'friction' describing the coupling between the two walls of the earthquake fault). At present, the most promising approaches to the understanding of the mechanism of this phenomenon include two classes of models, namely the crack model within a continuum (e.g., Madariaga and Cochard, 1996; Beeler and Tullis, 1996; Andrews and BenZion, 1997; Fukuyama and Madariaga, 1998) and the numerical implementations of the Burridge-Knopoff conceptual spring-block model (e.g., Burridge and Knopoff, 1967; Carlson and Langer, 1989; Vieira et al., 1993; Schmittbuhl et al., 1993; 1996; Espanol, 1994; Chen, 1996; Langer et al., 1996). In the spring-block model the most commonly used frictional constitutive relation is velocitydependent and the self-healing slip pulse is suggested to be related to the propagation of a solitary wave (e.g., Schmittbuhl et al., 1993; 1996; Espanol, 1994).

In the perspective of the solitary wave model, an interesting question is how strongly the existence of a solitary wave solution depends on the constitutive law of rock friction. Theoretically, this question is related to the fact that knowledge of rock friction under the conditions of earthquake sources is still not complete (see, e.g., Scholz, 1990,1998 ), resulting in difficulties in understanding the self-healing slip pulse in the perspective of rock mechanics. Practically, this question is related to the fact that observation seems to show that the existence of the selfhealing slip pulse is a common characteristic of large earthquakes (Heaton, 1990), from interplate earthquakes (e.g., Wald and Heaton, 1994) to intraplate (continental) earthquakes (e.g., Xu et al., 1997), occurring within different seismogenic environments in which there is no reason to require that the constitutive laws of rock friction are the same. To investigate this problem, in this paper, we consider an alternative type of frictional constitutive law, i.e., the slip-dependent one, to explore to what extent the existence of a self-healing slip pulse may tolerate the variation of the type of coupling between the two walls of the earthquake fault. Also, we take an alternative approach other than numerical calculations, i.e., we try to give an analytical solution. The analytical solution, being very simple, may be of help in the understanding of some of the properties of the self-healing slip pulse. Also, it may help to avoid limitation at the boundary due to the finite size of the numerical model. 


\section{The one-dimensional model for earthquake faulting}

We start with a scaled one-dimensional model for earthquake faulting (Carlson and Langer, 1989; Knopoff et al., 1992; Xu and Knopoff, 1994) described by

$$
\ddot{u}=c_{0}^{2} \frac{\partial^{2} u}{\partial x^{2}}-F(w, u, \dot{u})
$$

in which $u=u(x, t)$ is the slip along the fault at position $x$ and time $t$. In equation (1), the first term on the right hand side describes the elasticity and the second term describes the 'friction', including the stick-slip friction in the purely mechanical sense, the dissipation of elastic energy through the radiation of seismic waves and the coupling between the two walls of the earthquake fault; we will use the word 'friction' in this sense for simplicity. The form of function $F(w, u, \dot{u})$ depends on the understanding of the mechanisms of the processes as stated above. This model may be regarded as a continuum limit of the Burridge-Knopoff conceptual spring-block model (e.g., Burridge and Knopoff, 1967), in which $w$ is the loading velocity. Carlson and Langer (1989), Vieira et al.(1993), Schmittbuhl et al. (1993) and especially Espanol (1994) studied the situation in which $F(w, u, \dot{u})$ includes two terms: the linear coupling term $k u$, and the velocity-weakening friction $\phi(\dot{u})$; they found that for some combination of parameters there exists a solitary wave solution to the model earthquake faulting. We consider an alternative situation where $w \approx 0$ (slow loading) and 'friction' is slip-dependent. In this case equation (1) becomes

$$
\ddot{u}-c_{1)}^{2} \frac{\partial^{2} u}{\partial x^{2}}+V^{\prime}(u)=0
$$

in which

$$
V^{\prime}(u) \equiv \frac{d V(u)}{d u}
$$

\section{Solitary wave solutions}

Equation (1), being referred to as the non-linear KleinGordon equation, has a solitary wave solution for certain forms of function $V(u)$. Considering

$$
u(x, t)=u(\xi)
$$

in which

$$
\xi=x-c t
$$

where $c$ is a constant, one has

$$
\left(c^{2}-c_{n}^{2}\right) \frac{\partial^{2} u}{\partial \xi^{2}}+V^{\prime}(u)=0
$$

Defining

$$
v \equiv \frac{\partial u}{\partial \xi}
$$

Equation (3) is easily written as a pair of the first-order differential equations

$$
\left\{\begin{array}{l}
\frac{\partial u}{\partial \xi}=v \\
\frac{\partial v}{\partial \xi}=-\frac{V^{\prime}(u)}{c^{2}-c_{0}^{2}}
\end{array}\right.
$$

Its equilibrium points $\left(u_{0}, v_{0}\right)$ are determined by

$$
\begin{gathered}
v_{10}=0 \\
V^{\prime}\left(u_{01}\right)=0
\end{gathered}
$$

Its trajectory in the $(u, v)$-space may be determined by

$$
\frac{d v}{d u}=-\frac{V^{\prime}(u)}{\left(c^{2}-c_{0}^{2}\right) v}
$$

Integrating (7) leads to

$$
\frac{1}{2}\left(c^{2}-c_{n}^{2}\right) v^{2}+V(u)=A
$$

where $A$ is a constant.

$$
\text { From (5) }
$$

$$
\frac{\partial^{2} v}{\partial \xi^{2}}+\frac{V^{\prime \prime}(u)}{c^{2}-c_{\|}^{2}} v=0
$$

It is clear that when

$$
\frac{V^{\prime \prime}\left(u_{0}\right)}{c^{2}-c_{n}^{2}}>0
$$

there will be a periodic solution. On the other hand, as equation (5) has homoclinity or heteroclinity (as shown in Figure 1), equation (1) will have a solitary wave solution (see, e.g., Sagdeev et al., 1988; Liu and Liu, 1994). Espanol (1994) has systematically studied the situation of velocityweakening friction and found that in some regions of the parameter space the system exhibits periodic solutions, while for other regions the system has solitary wave solutions.
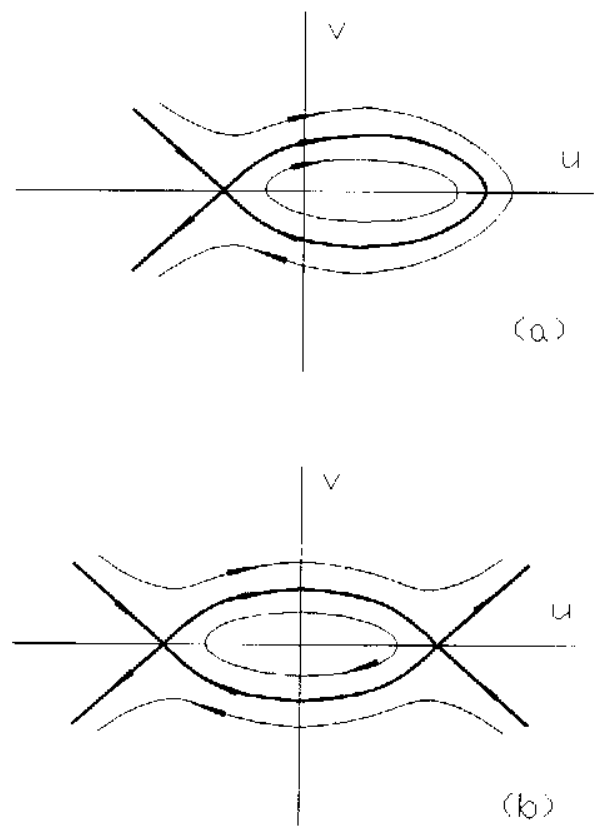

Fig.1 Schematic diagrams demonstrating examples of (a) Homoclinic trajectory (thick lines); (b) Heteroclinic trajectory (thick lines) 


\section{A special example}

As a special example, we use the scaled unit, and consider the first term of the Fourier expansion of function $V^{\prime}(u)$

$$
V^{\prime}(u)=f_{n}^{2} \sin u
$$

Such an approximation leads to a sine-Gordon equation. In our problem, a constraint that the rupture is irreversible i.e., $u \geq 0$, is imposed. Also from the experimental results (see, for example, Scholz, 1990), we have the constraint

$$
0 \leq u \leq \alpha \pi
$$

where $0<\alpha<1$. The physical significance of such an approximation is shown in Figure 2. We use this example to show how the system may sustain a solitary wave solution. Since we are considering the situation without any limitation on the number of blocks, we need not worry about the threshold condition caused by the boundary effects. As to the dissipative condition, in which the input energy is exactly balanced by the dissipation of the system, we find that the slip-dependent 'friction' is simpler to understand. In physics, the slip-dependent 'friction' corresponds to the coupling between the two walls of the earthquake fault caused by asperities and/or barriers with a certain kind of size distribution, e.g., power-law distribution. As the slip increases, there is an increasing probability to meet a larger barrier, leading to the increase of the coupling function. In this case, if we are considering a propagating crack with constant friction, we will find that the farther away from the crack tip the larger the slip, with which we are very familiar in the classical theory of a seismic source. On the other hand, as we consider the slip-dependent 'friction', the increase of slip will lead to the increase of the 'friction'. When the force driving the increase of the slip is exceeded by the 'friction', the slip stops. As a result, the slip is confined to a limited region, and the propagating rupture behaves as a solitary wave.

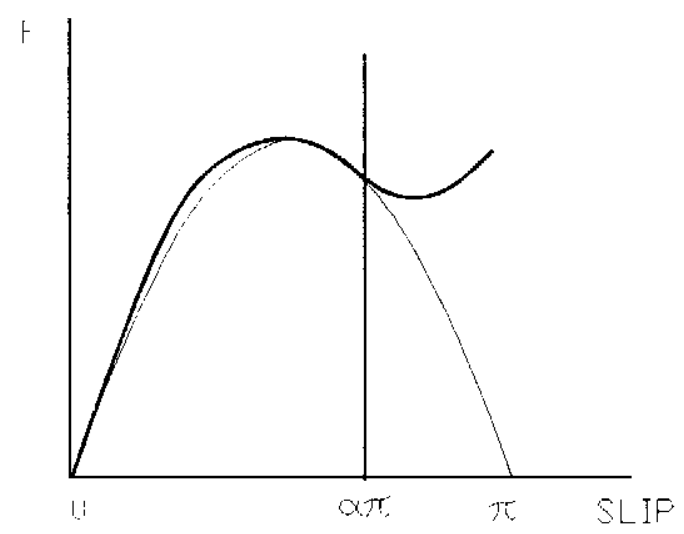

Fig.2 Schematic diagram demonstrating the physical significance of the first order approximation. See text for details. In the figure a scaled unit is used for the slip. The thick line describes the stip-dependent friction (from Scholz, 1990). The thin line gives the first order approximation. The slip is taken as one-sided (i.c., the rupture has no overshot) and below a certain value.
In this case, the equilibrium points of equations (5) may be determined by

$$
\begin{aligned}
& \sin u_{0}=0 \\
& v_{\mathrm{a}}=0
\end{aligned}
$$

and equation ( 8 ) becomes

$$
\frac{1}{2}\left(c^{2}-c_{11}^{2}\right)\left(\frac{\partial u}{\partial \xi}\right)^{2}-f_{0}^{2} \cos u=A
$$

As

$$
\cos u=1-2 \sin ^{2} \frac{u}{2}
$$

one has

$$
\frac{1}{2}\left(c^{2}-c_{0}^{2}\right)\left(\frac{\partial u}{\partial \xi}\right)^{2}+2 f_{0}^{2} \sin ^{2} \frac{u}{2}=B
$$

where

$$
B=A+f_{11}^{2}
$$

Considering a special situation that

$$
\begin{aligned}
& c<c_{0} \\
& B=0
\end{aligned}
$$

one has

$$
\frac{\partial u}{\partial \xi}= \pm \frac{2 f_{0}}{\sqrt{c_{0}^{2}-c^{2}}} \sin \frac{u}{2}
$$

Since

$$
\int \frac{d x}{2 \sin \frac{x}{2}}=\ln \tan \frac{x}{4}
$$

we have

$$
u(x, t)=4 \tan ^{-1}\left(\exp \left\{ \pm \frac{f_{11}}{\sqrt{c_{10}^{2}-c^{2}}}(x-c t)\right\}\right)
$$

which provides a solitary wave solution to the problem of earthquake faulting. In the standard solution of the sineGordon equation, it is the kink solution (for positive symbol) and the anti-kink solution (for negative symbol). Considering the physical significance of $u(x, t)$ we take the anti-kink solution. Also from constraint (12), we take the 'half-anti-kink' solution, as shown in Figure 3. In this case the slip behaves as a propagating solitary wave with velocity $c<c_{11}$, which may be of help to the understanding of the nature of the self-healing slip pulse. In Figure 3, taking $x$ as a constant, the 'half-anti-kink solution' shows the source time function at a certain position, which is similar to the Ben-Menaham-Toksoez model.

The result with a clear physical significance is that the width of the solitary wave $W$ may be estimated by

$$
W-\frac{\sqrt{c_{11}^{2}-c^{2}}}{f_{11}}
$$

implying that the width of the self-healing slip pulse depends on the friction and the rupture propagation velocity. The larger the friction, the narrower the pulse. As the friction becomes very large, the pulse disappears by $W \rightarrow 0$, which is consistent with experience in physics. This also agrees with the result of Espanol (1994) that the 
situation with a strong 'friction' (or equivalently, weak elasticity) corresponds to the single block limit. On the other hand, the nearer the rupture propagation velocity to the velocity of the seismic wave, the narrower the selfhealing slip pulse. When $c \rightarrow c_{0}$, the self-healing slip pulse vanishes by $W \rightarrow 0$, forming a physical barrier prohibiting the 'rupture velocity' from being supersonic.

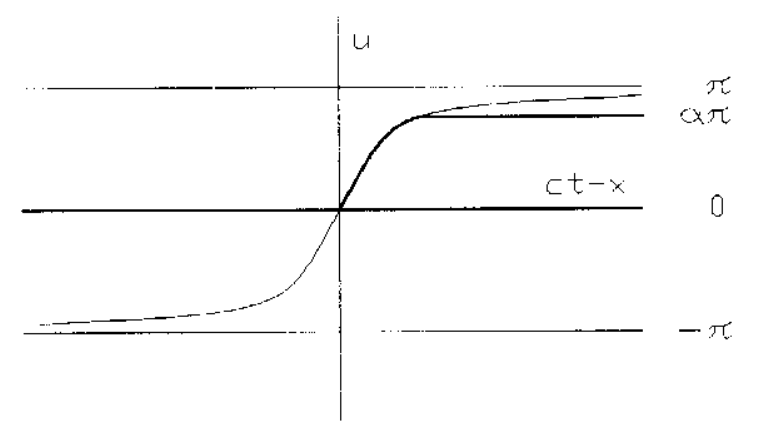

Fig.3 Schematic diagram describing the solitary wave solution to the sineGordon equation. The thin line indicates the standard anti-kink solution and the thick line indicates the 'half-anti-kink solution' corresponding to the solution of earthquake faulting.

\section{Discussion and Conclusions}

By studying a one-dimensional Burridge-Knopoff springblock model with slip-dependent frictional constitutive laws, we analytically explore the possibility of a solitary wave existing, as a feasible explanation of the self-healing slip pulse during earthquake rupture. When the frictional constitutive law has the form such that homoclinity or heteroclinity exists in the solution of equation (5), there will be a solitary wave solution to the problem of earthquake faulting. By taking the first order approximation, we discussed a special example in which a solitary wave solution exists. The slip behaves as a propagating solitary wave, with its velocity of propagation less than that of a seismic (acoustic) wave, and the source time function at each position remains the same, being similar to the BenMenaham-Toksoez model.

By nature, the Burridge-Knopoff spring-block model has an origin of continuum fracture mechanics (Rundle, 1991). However, the Burridge-Knopoff model addresses the overall properties of the earthquake faulting process. The role of the B-K model in earthquake dynamics is just like the lsing model and/or percolation model in statistical mechanics. In observation, the self-healing slip pulse behaves as a universal, overall property of earthquake faulting. In this sense, the spring-block model is useful in the description of overall properties far away from the edge of the fracture, especially the crack tip; this is useful in the understanding of the nature of large earthquakes in which the overall dimension of the seismic source is much larger than the size of the 'process zone' near to the crack tip. For the same reason, the present model is not able to provide the details of how the earthquake rupture organizes itself into a self-healing slip pulse, although the model shows that there exists a self-healing slip pulse solution (the solitary wave solution) to the problem.

In the spring-block model, the self-healing slip pulse may be explained as the propagation of a kind of solitary wave (e.g., Schmittbuhl et al., 1993; 1996; Espanol, 1994). A characteristic of such an idea is that, in other models, the rupturing and the healing are generally considered as two relatively independent processes while, in this model, the rupturing and the healing are considered as the components of a common physical process, in which the width of the Heaton pulse has clear physical significance, providing the earthquake catalogue with another useful source parameter. The common outstanding problem associated with the idea of solitary wave is that, generally, higher modes of the solitary wave, which are usually weaker and slower than the preliminary mode, may also exist. Present models consider only the preliminary mode, as in this paper. At present, the existence of the higher modes cannot be resolved due to the limitations on the resolution of seismic inversion technique. Testing the idea of a solitary wave will depend on enhancement of the resolution of seismic observation and interpretation.

In the present paper the idea of a solitary wave as a clue to understanding the mechanism of the self-healing slip pulse is the same as that in previous works, especially that of Schmittbuhl et al. (1993, 1996) and Espanol (1994); meanwhile, some alternative approaches have been undertaken. First, we take an analytical rather than numerical approach to the problem. The analytical solution, being very simple, may provide some simple and direct understanding of some of the properties of the self-healing slip pulse. We found from the model that the velocity of propagation of the self-healing slip pulse is less than the velocity of the acoustic (seismic) wave. It should be pointed out that our model does not forbid the existence of supersonic rupture propagation, only that the supersonic state cannot be reached by increasing the velocity of rupture propagation from the state of $c<c_{i j}$. The dependence of the pulse width on the strength of the friction is also consistent with experience in physics. Secondly, we consider a slipdependent frictional constitutive law to explore whether a solitary wave solution may exist for situations other than the velocity-dependent fricticnal constitutive laws. We take this approach because theoretically knowledge of rock friction under the condition of earthquake sources is still not complete (see, e.g., Scholz, 1990, 1998) and, in observation, it seems that the existence of the self-healing slip pulse is a common characteristic of large earthquakes (Heaton, 1990), from interplate earthquakes (e.g., Wald and Heaton, 1994) to intraplate (continental) ones (e.g., Xu et al., 1997), occurring within different seismogenic environments in which there is no reason to require that the constitutive laws of rock friction are the same. So an interesting question is, how strongly does the existence of a solitary wave solution to the problem of earthquake faulting depend on the constitutive law of rock friction. We consider this problem only for a special alternative case, i.e., slip-dependent 
coupling, which is by no means superior to its competing models, e.g., the velocity-weakening model, which is commonly cited, and the slip-gradient-dependent model which has not been fully studied so far. In future studies, it will be necessary to consider more realistic coupling constitutive laws. But, without losing generality, the implications of our result is double-sided. The pessimistic implication is that study the behavior of the self-healing slip pulse is not sufficient to determine the type of coupling constitutive laws along the earthquake fault, at least based on the present resolution of seismic observation. The optimistic implication is that the explanation of the selfhealing slip pulse may not require detailed knowledge of the coupling constitutive law as well as the friction.

Acknovledgments. We would like to thank Professor L. Knopoff of IGPP, UClA for constructive suggestions to improve the manuscript, and Professor Gu Hao-ding of CSB and Professor Liu Shi-da of Peking University for helpful discussion. This work is supported by NSFDYS Grant 49725410, also partly supported by CSB Project 95-07-411.

\section{References}

Andrews. D. J. and Ben-Zion, Y., Wrinkle-like slip pulse on a fault between different materials, J. Geophys. Res., 102. 553-571, 1997.

Becler, N. M. and Tullis, T. E.. Self-healing slip pulses in dynamic rupture models duc to velocity-dependent strength, Bull. Seism. Soc. Amer. 86. 1.130-1,148, 1996.

Burridge, R. and Knopolr, L., Model and theoretical scismicity, Bull. Seism. Soc: Amer., 57, 341-371, 1967.

Carlson, J. M. and Langer, J. S.. Mcchanical model of an earthquake fault, Phys Rev. A40, 6.470-6,484, 1989

Chen. X. X.. $\wedge$ two-dimensional earthquake fault modeling with fractal structure strength distribution. Acta Seismologica Sinica, English Edition. 9, 593-603, 1996.

Espanol, P.. Propagative slipping modes in a spring-block model, Phys. Rev, E50. 227-235, 1994.

Fukuyama. F. and Madariaga, R., Rupture dynamics of a planar fault in a 3-D elastic medium: rate- and slip-weakening friction, Bull. Seism. Soc. Amer.. 88, 1-17, 1998.

Heaton. T. H., Evidence for and implications of self-healing pulses of slip in carthquake rupture, Phys. Earth Planet. Interi., 64, 1-20, 1990.

Ide, $S$. and Takeo, M., Determination of constitutive relations of fault slip based on seismic wave analysis, J. Geophys. Res., 102, 27,379-27,391, 1997.
Irikura, K., Iwata, T., Sekiguchi, H., Pitarka, A. and Kamae, K., Lesson from the 1995 Hyogo-Ken Nanbu earthquake: why were such destructive motions generated to buildings? J. Natural Disaster Sci, 17, 99-127, 1996.

Knopoff, L., Landoni, J. A. and Abinantc, M. S., Dynamical model of an earthquake fault with localization, Phys. Rev., A46, 7,445-7,449, 1992.

Langer, J. S., Carlson, J. M., Myers, C. R. and Shaw, B. E., Slip complexity in dynamic models of earthquake faults, Proc. Natl. Acad. Sci. USA, 93, 3,825-3,829, 1996.

Liu, S. D. and Liu, S. K., Solitary wave and turbulence, Shanghai Scientific and Technological Education Publishing House, Shanghai, in Chinese, 1994.

Madariaga, R. and Cochard, A., Dynamic friction and the origin of the complexity of earthquake sources, Proc. Natl. Acad. Sci. USA, 93, 3,819-3,824, 1996

Nakayama, W. and Takeo, M., Slip history of the 1994 Sanriku-HarukaOki, Japan, earthquake deduced from strong motion data, Bull. Seism. Soc. Amer., 87, 918-931, 1997

Rundle, J. B., The statistical mechanics of carthquakes, Lecture notes, Workshop on nonlinear dynamics and earthquake prediction, Trieste, Italy, 1991; Chinese Translation (by Wu, Z. 1.): Translated World Seismology, (1), 80-88; (2), 82-88, 1994.

Schmittbuhl, J., Vilotte, J.-P. and Roux, S., Propagative macrodislocation modes in an earthquake fault model, Europhys. Lett., 21, 375-380, 1993.

Schmittbuhl, J., Vilotte, J.-P. and Roux, S., A dissipation-based analysis of an earthquake fault model, J. Geophys. Res., 101, 27,741-27,764. 1996.

Scholz, C. H., The mechanics of earthquakes and faulting, Cambridge Univ. Press, Cambridge. 1990.

Scholz, C. H., Earthquakes and friction laws, Nature, 391, 37-42, 1998.

Vicira, M. de S., Vasconcelos, G. L. and Nagel, S. R., Dynamics of spring-block modcls: tuning to criticality, Phys. Rev., E47, 2,221-2,224, 1993.

Wald, D. J. and Heaton, T. H., Spatial and temporal distribution of slip for the 1992 Landers, California, earthquake, Bull. Seism. Soc. Amer., 84. 668-691, 1994.

$\mathrm{Xu}, \mathrm{H}$ - J. and Knopoff, L., Periodicity and chaos in a onc-dimensional dynamical model of carthquakes, Phys. Rev., E50, 3,577-3,581, 1994.

Xu, I. S., Chen, Y. T. and Fasthoff, S., Temporal and spatial complexity in the rupture process of the February 3, 1996. Lijiang. Yunnan, China, earthquake. In: Chen, Y. T. (Ed.), Developments in Chinese seismology: congratulations to the 80th birthday of Professor Xie Yushou, Scismological Press, Beijing, 91-105, in Chinese with English abstract. 1997.

Sagdeev, R. Z., Usikov, D. A. and Zaslavsky, G. M., Nonlinear physics: from the pendulum to turbulence and chaos, Harwood Academic Publishers, Chur, 1988. 